\title{
Satisfacción laboral del personal de salud en una zona de conflicto armado de Cusco, Perú, 2013
}

\author{
Job satisfaction of healthcare workers in an armed conflict zone, \\ Cusco, Peru, 2013
}

\author{
Víctor Vera-Monge ${ }^{1, a}$, Carmen Inga-Chávez ${ }^{2, b}$, Noé Atamari-Anahui ${ }^{3, c}$ \\ Hospital Clínico de Salamanca, España. \\ ${ }^{2}$ Facultad de Enfermería, Universidad Nacional San Antonio Abad del Cusco, Perú. \\ ${ }^{3}$ Facultad de Medicina Humana, Universidad Nacional San Antonio Abad del Cusco, Perú. \\ ${ }^{a}$ Médico residente de neurologia; ${ }^{\mathrm{b}}$ Licenciada en enfermeria; ${ }^{\circ}$ Estudiante de Medicina.
}

An Fac med. 2015;76(3):289-90 / http://dx.doi.org/10.15381/anales.v76i3.11242

\section{SR. EDITOR:}

La satisfacción laboral es definida como un estado emocional positivo o placentero resultante de una percepción subjetiva de las experiencias laborales del sujeto ${ }^{(1)}$. La satisfacción del trabajador en el desempeño de sus funciones cumple un papel muy importante como determinante de la calidad de atención en salud; por ello la medición de la satisfacción laboral constituye una tarea necesaria.

En el Perú se tiene limitada información sobre la satisfacción laboral que presenta el personal de salud en algunas zonas que experimentaron un conflicto armado, siendo una de ellas el sector de Kiteni del distrito de Echarate (colindante al VRAE - valle de los ríos Apurímac y Ene) de la Provincia de la Convención en la Región del Cusco ${ }^{(2)}$.

Se realizó un estudio descriptivo, transversal entre los meses de marzo y abril del 2013, para establecer el nivel de satisfacción laboral de los trabajadores de algunos de los establecimientos de la microred de servicios de salud de Kiteni del Ministerio de Salud. La población de estudio estuvo conformada por el personal de salud que labora en dicha zona. Se realizó muestreo por conveniencia obteniéndose un total de 28 trabajadores, los cuales aceptaron voluntariamente participar del estudio. La satisfacción laboral se evaluó mediante el "Cuestionario de satisfacción laboral S10/12" desarrollado por Meliá y Pieró, una versión resumida del test validado por dichos autores, que se eligió por su corta extensión y una consistencia interna aceptable (alfa de Cronbach $=0,88)^{(3)}$. Dicho cuestionario está compuesto por 12 ítems que utiliza 7 grados de alternativas: insatisfecho de 1 a 3 ; indiferente 4 y satisfecho de 5 a 7 . El cuestionario fue anónimo y autoadministrado.

De los 28 trabajadores de salud encuestados 10 eran varones y 18 mujeres. Según el cargo que desempeñaban se encontró que 22 eran del personal asistencial, 3 del personal administrativo, 2 jefes de establecimientos y un trabajador como personal de servicio. En cuanto a la profesión, 4 eran médicos, 10 del personal de enfermería, 7 eran obstetrices y 3 odontólogos; además de 4 agrupados en la categoría de otros. Según la edad de los trabajadores, 13 tenían edad menor de 30 años, 11 entre 30 y 40 años y solo 4 eran mayores de 40 años. La condición laboral que presentaban fue la siguiente: 4 trabaja- dores como personal de SERUMS (servicio rural y urbano marginal de salud), 20 en condición de CAS (Contrato Administrativo de Servicios) y 4 como personal nombrado.

Según los diferentes ítems del cuestionario se obtuvieron los siguientes resultados (tabla 1). El 54\% $(n=15)$ del personal de salud se mostró insatisfecho con el entorno físico, un 50\% $(n=14)$ con el cumplimiento laboral por parte de esta y $43 \%(n=12)$ con el trato de la empresa. Un 64\% $(n=18)$ del personal de salud estuvo satisfecho con las relaciones personales con sus superiores, un $47 \%(n=13)$ con la temperatura ambiental, 54\% $(n=15)$ con la proximidad, frecuencia y forma de estas, $64 \%(\mathrm{n}=18)$ con las supervisiones y el $50 \%(n=14)$ mostró satisfacción con el apoyo de sus superiores. En cuanto a los objetivos y metas, higiene y forma de negociación laboral con la empresa, no se encontró diferencia significativa con el nivel de satisfacción laboral (insatisfecho o satisfecho), destacando un cierto nivel de indiferencia por parte del personal de salud.

Estos hallazgos nos muestran que el laborar en una zona de conflicto armado, podría alterar en cierta medida el 
Tabla 1. Frecuencias y porcentajes según cada ítem del cuestionario de satisfacción laboral S10/12 aplicado al personal de salud de la microred de servicios de salud de Kiteni (La Convención, Cusco-Perú).

\begin{tabular}{|c|c|c|c|c|c|c|c|c|c|c|c|c|c|c|c|c|c|c|}
\hline \multirow{3}{*}{ ENUNCIADO* } & \multicolumn{6}{|c|}{ Insatisfecho } & \multicolumn{10}{|c|}{ Satisfecho } & \multirow{2}{*}{\multicolumn{2}{|c|}{ Total }} \\
\hline & \multicolumn{2}{|c|}{ Muy } & \multicolumn{2}{|c|}{ Bastante } & \multicolumn{2}{|c|}{ Algo } & \multicolumn{2}{|c|}{ Total } & \multicolumn{2}{|c|}{ Indiferente } & \multicolumn{2}{|c|}{ Algo } & \multicolumn{2}{|c|}{ Bastante } & \multicolumn{2}{|c|}{ Muy } & & \\
\hline & $\mathrm{N}$ & $\%$ & $\mathrm{~N}$ & $\%$ & N & $\%$ & $\mathrm{~N}$ & $\%$ & $\mathrm{~N}$ & $\%$ & $\mathrm{~N}$ & $\%$ & $\mathrm{~N}$ & $\%$ & N & $\%$ & $\mathrm{~N}$ & $\%$ \\
\hline $\begin{array}{l}\text { Los objetivos, metas y tasas de } \\
\text { producción que debe alcanzar }\end{array}$ & 1 & 4 & 7 & 25 & 4 & 14 & 12 & 43 & 4 & 14 & 6 & 21 & 6 & 21 & 0 & 0 & 12 & 42 \\
\hline $\begin{array}{l}\text { La limpieza, higiene y salubridad de su } \\
\text { lugar de trabajo }\end{array}$ & 5 & 18 & 4 & 14 & 4 & 14 & 13 & 46 & 3 & 11 & 5 & 18 & 7 & 25 & 0 & 0 & 12 & 43 \\
\hline $\begin{array}{l}\text { El entorno físico y el espacio de que } \\
\text { dispone en su lugar de trabajo }\end{array}$ & 5 & 18 & 3 & 11 & 7 & 25 & 15 & 54 & 3 & 11 & 6 & 21 & 3 & 11 & 1 & 4 & 10 & 36 \\
\hline La temperatura de su local de trabajo & 4 & 14 & 3 & 11 & 3 & 11 & 10 & 36 & 5 & 18 & 10 & 36 & 1 & 4 & 2 & 7 & 13 & 47 \\
\hline $\begin{array}{l}\text { Las relaciones personales con sus } \\
\text { superiores }\end{array}$ & 2 & 7 & 2 & 7 & 4 & 14 & 8 & 28 & 2 & 7 & 11 & 39 & 5 & 18 & 2 & 7 & 18 & 64 \\
\hline La supervisión que ejercen sobre usted & 1 & 4 & 2 & 7 & 3 & 11 & 6 & 22 & 4 & 14 & 11 & 39 & 6 & 21 & 1 & 4 & 18 & 64 \\
\hline $\begin{array}{l}\text { La proximidad y frecuencia con que es } \\
\text { supervisado }\end{array}$ & 1 & 4 & 2 & 7 & 4 & 14 & 7 & 25 & 6 & 21 & 10 & 36 & 5 & 18 & 0 & 0 & 15 & 54 \\
\hline $\begin{array}{l}\text { La forma en que sus supervisores juzgan } \\
\text { su tarea }\end{array}$ & 3 & 11 & 3 & 11 & 4 & 14 & 10 & 36 & 3 & 11 & 12 & 43 & 3 & 11 & 0 & 0 & 15 & 54 \\
\hline $\begin{array}{l}\text { La "igualdad" y "justicia" de trato que } \\
\text { recibe de su empresa }\end{array}$ & 4 & 14 & 3 & 11 & 5 & 18 & 12 & 43 & 7 & 25 & 7 & 25 & 2 & 7 & 0 & 0 & 9 & 32 \\
\hline El apoyo que recibe de sus superiores & 2 & 7 & 2 & 7 & 5 & 18 & 9 & 32 & 5 & 18 & 9 & 32 & 4 & 14 & 1 & 4 & 14 & 50 \\
\hline $\begin{array}{l}\text { El grado en que su empresa cumple } \\
\text { el convenio, las disposiciones y leyes } \\
\text { laborales }\end{array}$ & 7 & 25 & 3 & 11 & 4 & 14 & 14 & 50 & 2 & 7 & 10 & 36 & 2 & 7 & 0 & 0 & 12 & 43 \\
\hline $\begin{array}{l}\text { La forma en que se da la negociación en } \\
\text { su empresa sobre aspectos laborales }\end{array}$ & 6 & 21 & 3 & 11 & 3 & 11 & 12 & 43 & 4 & 14 & 9 & 32 & 2 & 7 & 1 & 4 & 12 & 43 \\
\hline
\end{tabular}

*Nivel de satisfacción laboral en base a los ítems del cuestionario: Satisfacción con el ambiente físico (items 1 al 4), satisfacción con la supervisión (ítems 5 al 10) y satisfacción con las prestaciones recibidas (ítems del 11 al 12).

nivel de satisfacción laboral del personal de salud de atención primaria, e incluso ser un factor de riesgo para la vulneración de la seguridad del personal de salud ${ }^{(4)}$, lo que se convierte en un peligro si no se cuenta con información al respecto, lo cual podría repercutir en la prestación de servicios de salud ${ }^{(5)}$. En nuestro estudio se evidenció que los aspectos como el ambiente físico de trabajo y las prestaciones recibidas fueron los que mostraron algún grado de insatisfacción, posiblemente por el estrés psicológico que genera el estar cerca a las zonas de enfrentamiento armado y otros factores que pueden contribuirla ${ }^{(6)}$. Por ello recomendamos tomarlo en consideración, pues el conocerlo hace que sea una fuente de información que ayudaría a las autoridades del sector salud para la formulación de políticas relacionadas al mejoramiento de condiciones laborales de los recursos humanos y más aún en atención primaria ${ }^{(7)}$, lo que conduciría a mejorar la calidad de los servicios de salud brindado en las zonas de conflicto armado. De esta forma, hacemos énfasis en que se debe fomentar la realización de estudios con mejor metodología y mayor alcance sobre la satisfacción laboral en el personal de salud en áreas de conflicto armado.

\section{REFERENCIAS BIBLIOGRÁFICAS}

1. Locke EA. The nature and causes of job satisfaction. En: Dunnette MD (ed). Handbook of Industrial and Organizational Psychology. Chicago, IL: Rand McNally. 1976:1297-349.

2. Arce G. Los riesgos de militarizar la lucha contra el narcotráfico: algunos apuntes sobre el Plan VRAE. Coyuntura: Análisis Económico y Social de Actualidad Centro de investigaciones sociológicas, económicas, políticas y antropológicas - PUC Perú. 2008;4(17):18-21.

3. Melía JL, Peiró JM. El cuestionario de satisfacción S10/12: estructura factorial, fiabilidad y validez. Rev Psicol Trab Org. 1989;4(11):179-87.

4. Adil M, Johnstone P, Furber A, Siddiqi K, Khan D. Violence against public health workers during armed conflicts. The Lancet. 2013;381(9863):293. doi: 10.1016/S0140-6736(13)60127-0.
5. Atamari-Anahui NI, Sucasaca-Rodriguez C, Huamanquispe-Quintana J. Violencia contra el personal de salud: ¿Un problema silencioso en Perú? Rev cuerpo méd HNAAA. 2013;6(4):59.

6. Rodriguez-Marin J. Calidad de vida laboral en profesionales de la salud. Rev Calid Asist. 2010;25(6):318-20.

7. Garcia Cabrera HE, Diaz Urteaga P, Ávila Chávez D, Cuzco Ruiz MZ. La Reforma del Sector Salud y los recursos humanos en salud. An Fac med. 2015;76(SPE):7-26. doi:10.15381/anales. v76i1.10966.

Carta al Editor recibida para publicación el 14 de abril de 2015

\section{Fuentes de financiamiento: Autofinanciado.}

Conflictos de interés: WW y $\mathrm{ClCH}$ han realizado el SERUMS (Servicio Rural y Urbano Marginal de Salud) en el Centro de Atención Primaria I de Echarate de EsSalud (Cusco, Perú) en el periodo 2012-2013.

\section{Correspondencia:}

Noé Atamari Anahui

Dirección: Calle Los Geranios B-2 San Sebastián, Cusco, Perú

Teléfono: 950383376

Correo electrónico: noe.atamari@gmail.com 